Pacific Journal of Mathematic 


\title{
INTRINSIC MEASURES AND HOLOMORPHIC RETRACTS
}

\author{
IAN GRAHAM
}

In this paper we examine the consequences of the equality of the Eisenman and Carathéodory norms on $k$-vectors, $2 \leq k \leq n-1$, at a point in an $n$-dimensional complex manifold $M$. We also investigate the consequence of the existence of a large number of two-dimensional holomorphic retracts of a complex manifold-one tangent to each 2-vector at a given point.

1. Introduction. The equality of the Kobayashi and Carathéodory metrics on strictly convex domains in $\mathbf{C}^{n}$ is one consequence of the work of Lempert $[12,13,14,19]$. This remarkable fact is deduced from the existence of a large supply of one-dimensional holomorphic retracts of such domains-one through each point tangent to each direction. In this paper we examine the consequences of the equality of the Eisenman and Carathéodory norms on $k$-vectors, $2 \leq k \leq n-1$, at a point in an $n$-dimensional complex manifold $M$. (The Eisenman norm is the analog of the infinitesimal Kobayashi metric and is the object of a recent study by $\mathrm{H}$. Wu and the author [9]; see also the papers of Eisenman $[7,15]$.) $\mathrm{H}$. Wu and the author considered the top-dimensional case in [10] (for earlier results see $[6,17,22]$ ) obtaining a criterion for biholomorphic equivalence with the unit ball in $\mathbf{C}^{n}$. We also investigate the consequences of the existence of a large number of two-dimensional holomorphic retracts of a complex manifold-one tangent to each 2-vector at a given point. Both assumptions lead to the conclusion, if $M$ is Carathéodory-hyperbolic (see $\S 4$ for precise statements of the theorems) that the indicatrix of the Carathéodory metric at the point in question is an analytic ellipsoid; the first assumption also implies that the infinitesimal Kobayashi and Carathéodory metrics coincide at the point in question. Later in the paper we consider intrinsic norms on $k$-vectors at the origin of a circular domain in $\mathbf{C}^{n}$, exhibiting some differences between the $k=1$ case and the $k>1$ case.

Lempert [13] has already observed that there do not in general exist 2-dimensional holomophic retracts containing three given points of a strictly convex domain; his argument shows that there does not in general exist a 2-dimensional retract tangent to a given 2-vector at a point. It is 
worth pointing out that some facts from the theory of Banach and Hilbert spaces shed some light on this situation and on the theorems proved here. Specifically, if $X$ is a real or complex Banach space, there always exists a projection of norm 1 onto any one-dimensional subspace by the HahnBanach theorem. However, if there exists a projection of norm 1 onto all two-dimensional subspaces, then the given Banach space $X$ is actually a Hilbert space. (The real case of this result was proved by Kakutani [11] and Phillips [16], the complex case by Bohnenblust [3].) Of course a projection of norm 1 is just a linear retraction of the unit ball of $X$, and, in the finite-dimensional case, $X$ is a Hilbert space iff the unit ball is an ellipsoid.

The plan of the paper is as follows: In $\S 2$ we recall the definitions of the Eisenman and Carathéodory norms on decomposable $k$-vectors. In $\S 3$ we give estimates in the form of two lemmas for these norms which are used in the proof of the theorems as well as in some further results in $\S 6$. The main theorems are proved in $\$ 4$. In $\$ 5$ we give a variant on the known versions of the Schwarz lemma on circular domains; this result as well as the lemmas of $\S 3$ are used in $\S 6$ to give further formulas and estimates for the Eisenman and Carathéodory norms at the origin of circular domains.

It is a pleasure to record my thanks to Man-Duen Choi and Peter Rosenthal for discussions about projections in Banach spaces and for locating the characterizations of Hilbert space by Kakutani [11] and Bohnenblust [3].

2. Definitions. In this section we recall some basic definitions, most of which appear in [9]. Let $M$ be an $n$-dimensional complex manifold and let $p \in M$. Let $T_{p} M$ (respectively $T M$ ) denote the holomorphic tangent space to $M$ at $p$ (respectively the holomorphic tangent bundle). Let $\Lambda^{k} T_{p} M$ (respectively $\Lambda^{k} T M$ ) be the $k$ th exterior power of $T_{p} M$ (respectively $T M$ ). Of special interest are the decomposable elements of $\Lambda^{k} T_{p} M$ (respectively $\Lambda^{k} T M$ ); these we denote by $D_{p}^{k} M$ (respectively $\left.D^{k} M\right)$. There is a natural way of extending a hermitian metric $\langle$,$\rangle on T M$ to a hermitian metric on $\Lambda^{k} T M$ : first, for $\alpha, \beta \in D_{p}^{k} M$ with $\alpha=v_{1} \wedge \cdots \wedge v_{k}$ and $\beta=w_{1} \wedge \cdots \wedge w_{k}$ we define

$$
\langle\alpha, \beta\rangle=\operatorname{det}\left(\left\langle v_{l}, w_{j}\right\rangle\right)_{l, j=1, \ldots, k}
$$

and then extend the definition to arbitrary elements of $\Lambda^{k} T_{p} M$ by linearity.

The unit ball in $\mathbf{C}^{n}$ is denoted by $B_{n}$. $B_{n}$ carries the Euclidean metric which may be extended to $k$-vectors as above. We orient $\mathbf{C}^{n}$ so that the $(n, n)$-form $(i / 2)^{n} d z_{1} \wedge d \bar{z}_{1} \wedge \cdots \wedge d z_{n} \wedge d \bar{z}_{n}$ is positive. 
For decomposable $k$-vectors, we define the Eisenman and Carathéodory norms by constructions modelled on the definitions of the differential Kobayashi and Carathéodory pseudo-metrics. Let $1 \leq k \leq n$ and let $\alpha \in D_{p}^{k} M$.

Definition 1. The Eisenman norm of $\alpha$ is

$$
E_{k}(p ; \alpha)=\inf \left\{\|\gamma\|^{2} \mid \gamma \in D_{0}^{k} B_{k}\right. \text { and there exists a holo- }
$$

(2.2) morphic mapping $f: B_{k} \rightarrow M$ such that $f(0)=p$ and $\left.f_{*}(\gamma)=\alpha\right\}$.

Definition 2. The Carathéodory norm of $\alpha$ is

$$
C_{k}(p ; \alpha)=\sup \left\{\|\gamma\|^{2}: \quad \gamma \in D_{0}^{k} B_{k}\right. \text { and there exists a }
$$

(2.3) holomorphic mapping $g: M \rightarrow B_{k}$ such that $g(p)=0$ and $\left.g_{*}(\alpha)=\gamma\right\}$.

By $\|\gamma\|$ we mean the Euclidean norm of $\gamma$; the reason for squaring (as in $[2,9,10])$ arises from the computation of volumes in local holomorphic coordinates. It suffices to define $E_{k}$ and $C_{k}$ for decomposable $k$-vectors in order to compute volumes. If $A$ is a $k$-dimensional complex submanifold of an open subset $U$ of $M$ we introduce intrinsic volume forms $\tau_{A}^{M}$ and $\eta_{A}^{M}$ as follows: let $p \in A$ and let $z_{1}, \ldots, z_{n}$ be local holomorphic coordinates in $M$ near $p$ so that $\partial / \partial z_{1}, \ldots, \partial / \partial z_{k}$ are tangent to $A$ at $p$. Then

$$
\tau_{A}^{M}(p)=E_{k}\left(p ; \frac{\partial}{\partial z_{1}} \wedge \cdots \wedge \frac{\partial}{\partial z_{k}}(p)\right)\left(\frac{i}{2}\right)^{k} d z_{1} \wedge d \bar{z}_{1} \wedge \cdots \wedge d z_{k} \wedge d \bar{z}_{k}
$$

is the Eisenman volume form of $A$ (as a submanifold of $M$ ) while

$$
\eta_{A}^{M}=C_{k}\left(p ; \frac{\partial}{\partial z_{1}} \wedge \cdots \wedge \frac{\partial}{\partial z_{k}}(p)\right)\left(\frac{i}{2}\right)^{k} d z_{1} \wedge d \bar{z}_{1} \wedge \cdots \wedge d z_{k} \wedge d \bar{z}_{k}
$$

is the Carathéodory volume form of $A$ (as a submanifold of $M$ ). If $S$ is a Borel subset of $A$ then its Eisenman volume is

$$
\mathscr{E}_{k}(S)=\int_{S} \tau_{A}^{M}
$$

and its Carathéodory volume is

$$
\mathscr{C}_{k}(S)=\int_{S} \eta_{A}^{M}
$$

When necessary we shall indicate the manifold on which the intrinsic $k$-norms are defined by a superscript, as in $E_{k}^{M}(;), C_{k}^{M}(;)$. It is elementary to verify that if $A$ is a complex submanifold of $M$ which is a 
holomorphic retract of $M$ then $E_{k}^{M}=E_{k}^{A}$ and $C_{k}^{M}=C_{k}^{A}$ on decomposable $k$-vectors of $A$. (There is a general theorem of Rossi to the effect that any holomorphic retract of a complex manifold must be a complex submanifold [18, Theorem 5.2].) Also the inequality $C_{k} \leq E_{k}$ is elementary [7].

The indicatrix of the Kobayashi pseudo-metric at $p \in M$ is the set $I_{K}(p)=\left\{v \in T_{p} M \mid E_{1}(p ; v)<1\right\} . E_{1}$ as defined above is actually the square of the Kobayashi pseudo-metric. Similarly $C_{1}$ is the square of the Carathéodory pseudo-metric so that the indicatrix of the Carathéodory pseudo-metric is the set $I_{C}(p)=\left\{v \in T_{p} M \mid C_{1}(p ; v)<1\right\}$. Both indicatrices are complete circular domains; $I_{C}(p)$ is convex.

By an analytic ellipsoid we shall mean a domain of the form

$$
\left\{z \in \mathbf{C}^{n} \mid \sum_{i, j=1}^{n} a_{i j} z_{i} \bar{z}_{j}<1\right\}
$$

where $\left(a_{\imath j}\right)_{l, j=1}^{n}$ is a positive definite hermitian matrix. Note that all such domains are centered at the origin.

Finally we recall that a complex manifold $M$ is said to be taut if for any other complex manifold $N$ the set of holomorphic mappings from $N$ into $M$ form a normal family [23].

3. Estimates for the Eisenman and Carathéodory norms. Let $p$ be a point in an $n$-dimensional complex manifold $M$ which carries a hermitian metric $\langle$,$\rangle . The unit ball B_{k}$ in $\mathbf{C}^{k}$ is equipped with the Euclidean metric. A preliminary estimate is given by the following:

Lemma 1 Let $\alpha \in D_{p}^{k} M$. Let $L$ be the $k$-dimensional (complex) linear subspace of $T_{p} M$ which is tangent to $\alpha$. Then

$$
\begin{aligned}
& E_{k}(p ; \alpha) \geq \frac{\|\alpha\|^{2} \operatorname{vol}\left(B_{k}\right)}{\operatorname{vol}\left(L \cap I_{K}(p)\right)}, \\
& C_{k}(p, \alpha) \leq \frac{\|\alpha\|^{2} \operatorname{vol}\left(B_{k}\right)}{\operatorname{vol}\left(L \cap I_{C}(p)\right)} .
\end{aligned}
$$

$\left(\|\alpha\|^{2}, \operatorname{vol}\left(L \cap I_{K}(p)\right)\right.$, and $\operatorname{vol}\left(L \cap I_{C}(p)\right)$ are computed with respect to the hermitian metric $\langle,\rangle ; \operatorname{vol}\left(B_{k}\right)$ denotes the Euclidean volume of the unit ball in $\mathbf{C}^{k}$. The right-hand sides of (3.1) and (3.2) are of course independent of the choice of hermitian metric on $M$.) 
Proof of (3.1) Suppose that $f: B_{k} \rightarrow M$ is a holomorphic map and $\gamma \in D_{0}^{k} B_{k}$ are such that $f(0)=p$ and $f_{*}(\gamma)=\alpha$. Then

$$
\frac{\left\|f_{*}(\gamma)\right\|^{2}}{\|\gamma\|^{2}}=\frac{\operatorname{vol}\left(f_{*}\left(B_{k}\right)\right)}{\operatorname{vol}\left(B_{k}\right)} \leq \frac{\operatorname{vol}\left(L \cap I_{K}(p)\right)}{\operatorname{vol}\left(B_{k}\right)}
$$

where the last inequality is a consequence of the fact that $f_{*}$ maps the indicatrix of the Kobayashi metric of $B_{k}$ at 0 into $L \cap I_{K}(p)$. (For the terms in the numerator of (3.3) the metric $\langle$,$\rangle is used, while the$ Euclidean metric is used for the terms in the denominator. Also by abuse of notation we are denoting the unit ball in $T_{0} B_{k}$ by $B_{k}$.) Thus

$$
\|\gamma\|^{2} \geq \frac{\|\alpha\|^{2} \operatorname{vol}\left(B_{k}\right)}{\operatorname{vol}\left(L \cap I_{K}(p)\right)}
$$

from which (3.1) follows.

Proof of (3.2) Suppose that $g: M \rightarrow B_{k}$ is a holomorphic map and $\gamma \in D_{0}^{k} B_{k}$ are such that $g(p)=0$ and $g_{*}(\alpha)=\gamma$. Then

$$
\frac{\left\|g_{*}(\alpha)\right\|^{2}}{\|\alpha\|^{2}}=\frac{\operatorname{vol}\left(g_{*}\left(L \cap I_{C}(p)\right)\right)}{\operatorname{vol}\left(L \cap I_{C}(p)\right)} \leq \frac{\operatorname{vol}\left(B_{k}\right)}{\operatorname{vol}\left(L \cap I_{C}(p)\right)}
$$

(Euclidean metric in the numerator, $\langle$,$\rangle in the denominator) from which$ (3.2) follows. As before we have used the fact that $g_{*}\left(I_{C}(p)\right) \subset B_{k}$.

The estimates in Lemma 1 can be sharpened. In fact we actually have

Lemma 2. Let $\alpha \in D_{p}^{k} M$. Let $L$ be the $k$-dimensional linear subspace of $T_{p} M$ which is tangent to $\alpha$.

(a) Let $s$ be the supremum of the volumes (w.r.t. $\langle\rangle$,$) of the analytic$ ellipsoids which can be inscribed in $L \cap I_{K}(p)$. (There is an ellipsoid of maximal volume if $L \cap I_{K}(p)$ is bounded.) Then

$$
E_{k}(p ; \alpha) \geq\|\alpha\|^{2} \operatorname{vol}\left(B_{k}\right) s^{-1} \text {. }
$$

(b) Let $t$ be the infimum of the volumes (w.r.t. $\langle$,$\rangle ) of the analytic$ ellipsoids which can be circumscribed about $L \cap I_{C}(p)$. ( $t$ is finite iff $L \cap I_{C}(p)$ is bounded, in which case there is a circumscribing ellipsoid of minimal volume.) Then

$$
C_{k}(p ; \alpha) \leq\|\alpha\|^{2} \operatorname{vol}\left(B_{k}\right) t^{-1} .
$$

(The estimates (3.6) and (3.7) are of course independent of the choice of $\langle$,$\rangle .)$ 
Proof (a) Referring to the proof of (3.1) in the previous lemma, we note that $f_{*}\left(B_{k}\right)$ must be an analytic ellipsoid and hence vol $\left(f_{*}\left(B_{k}\right)\right) \leq s$. This gives the sharpened estimate (3.6). To see that the supremum is attained if $L \cap I_{K}(p)$ is bounded, let $T_{j}: \mathbf{C}^{k} \rightarrow L$ be a sequence of complex-linear maps such that $T_{j}\left(B_{k}\right) \subset I_{K}(p)$ and $\operatorname{vol} T_{j}\left(B_{k}\right) \rightarrow s$. The maps $T_{j}$ are uniformly bounded, hence a subsequence converges in the norm topology to a linear map $T: \mathbf{C}^{k} \rightarrow T_{p} M$. Evidently $\operatorname{vol}\left(T\left(B_{k}\right)\right)=s$ and $T\left(B_{k}\right) \subset \overline{I_{K}(p)}$. But since $T$ is open we must have $T\left(B_{k}\right) \subset I_{K}(p)$.

(b) Referring to the proof of (3.2) in the previous lemma, we note that if there exist a holomorphic map $g: M \rightarrow B_{k}$ and $\gamma \in D_{0}^{k} B_{k}$ such that $g(p)=0$ and $g_{*}(\alpha)=\gamma$, then

$$
\frac{\left\|g_{*}(\alpha)\right\|^{2}}{\|\alpha\|^{2}}=\frac{\|\gamma\|^{2}}{\left\|g_{*}^{-1}(\gamma)\right\|^{2}}=\frac{\operatorname{vol}\left(B_{k}\right)}{\operatorname{vol}\left(g_{*}^{-1}\left(B_{k}\right)\right)} \leq \frac{\operatorname{vol}\left(B_{k}\right)}{t}
$$

since $g_{*}^{-1}\left(B_{k}\right)$ is an analytic ellipsoid which contains $L \cap I_{C}(p)$. (As the reader will surmise, by $g_{*}^{-1}$ we really mean $\left(\left.g_{*}\right|_{L}\right)^{-1}$.) The finiteness statement about $t$ is obvious, and the assertion that there is a circumscribing ellipsoid of minimal volume follows from applying a normality argument to the set of linear maps from $L$ to $C^{k}$ which map $L \cap I_{C}(p)$ into $B_{k}$.

\section{The main results.}

THEOREM 1. Suppose that $M$ is a complex manifold of dimension $n$ and $p \in M$. Suppose that for some integer $k, 1 \leq k \leq n$, there exists $\alpha \in D_{p}^{k} M$ such that $E_{k}(p ; \alpha)=C_{k}(p ; \alpha) \neq 0$. Let $L$ be the $k$-dimensional complex subspace of $T_{p} M$ which is tangent to $\alpha$. Then $E_{1}(p ; v)=C_{1}(p ; v)$ for all $v \in L$ and $I_{K}(p) \cap L=I_{C}(p) \cap L$ is an analytic ellipsoid.

Proof. The assumption that $C_{k}(p ; \alpha) \neq 0$ is easily seen to imply that $C_{1}(p ; v) \neq 0$ for all $v \in L-\{0\}$. The continuity of $C_{1}$ implies that $I_{C}(p)$ is bounded, hence $I_{K}(p)$ is bounded as well since $I_{K}(p) \subset I_{C}(p)$. By Lemma 2 there exist $k$-dimensional analytic ellipsoids $R \subset L \cap I_{K}(p)$ and $S \supset L \cap I_{C}(p)$ such that $R$ has maximal volume and $S$ has minimal volume, volumes being computed with respect to some hermitian metric $\langle$,$\rangle on M$ (the choice of which does not affect the choice of $R$ and $S$ ). Also by Lemma 2 we have

$$
E_{k}(p ; \alpha) \geq\|\alpha\|^{2} \frac{\operatorname{vol}\left(B_{k}\right)}{\operatorname{vol}(R)}
$$


and

$$
C_{k}(p ; \alpha) \leq\|\alpha\|^{2} \frac{\operatorname{vol}\left(B_{k}\right)}{\operatorname{vol}(S)} .
$$

These estimates, together with the assumption that $E_{k}(p ; \alpha)=C_{k}(p ; \alpha)$ and the obvious inequality $\operatorname{vol}(R) \leq \operatorname{vol}(S)$ lead us to the conclusion that $\operatorname{vol}(R)=\operatorname{vol}(S)$, from which it follows (since $R \subset S$ and both are analytic ellipsoids) that $R=I_{K}(p) \cap L=I_{C}(p) \cap L=S$.

THEOREM 2. With hypotheses as in Theorem 1, suppose that in addition $M$ is taut. Then there exists a $k$-dimensional complex submanifold $A$ of $M$ which is biholomorphic to $B_{k}$ and tangent to $\alpha$ at $p$, and there exists $a$ holomorphic retraction $\phi: M \rightarrow A$.

Proof. In view of the assumption of tautness of $M$, there exists a holomorphic map $f: B_{k} \rightarrow M$ and $\gamma_{1} \in D_{0}^{k} B_{k}$ such that $f(0)=p, f_{*}\left(\gamma_{1}\right)$ $=\alpha$, and $\left\|\gamma_{1}\right\|^{2}=E_{k}(p ; \alpha)$. Normality also implies that there exists a holomorphic map $g: M \rightarrow B_{k}$ and $\gamma_{2} \in D_{0}^{k} B_{k}$ such that $g(p)=0, g_{*}(\alpha)$ $=\gamma_{2}$, and $\left\|\gamma_{2}\right\|^{2}=C_{k}(p ; \alpha)$. By hypothesis $\left\|\gamma_{1}\right\|^{2}=\left\|\gamma_{2}\right\|^{2}$; hence since the fibre of $\Lambda^{k} T_{0} B_{k}=D_{0}^{k} B_{k}$ has dimension 1 we must have $\gamma_{2}=e^{i \theta} \gamma_{1}$ for some choice of $\theta$. By replacing $g$ by $e^{-\imath \theta} \cdot g$ we may assume that $\gamma_{1}=\gamma_{2}$. But this implies that the composed mapping $g \circ f: B_{k} \rightarrow B_{k}$ has Jacobian determinant equal to 1 at the origin and hence, since the origin is fixed, must be a unitary transformation $U$. By replacing $g$ by $U^{-1} \circ g$ we may assume that $g \circ f$ is the identity transformation.

Now let $A=f\left(B_{k}\right)$. It is easy to see that $A$ is a holomorphic retract of $M$ and in fact the map $\phi=f \circ g$ gives the desired retraction. The mapping $f$ must have maximal rank everywhere and be 1-1 since these statements are true of $g \circ f$. Hence $f$ is an embedding, which completes the proof. (We note that a theorem of Rossi [18, Theorem 5.2] guarantees that any holomorphic retract of a complex manifold must be a complex submanifold.)

REMARK. It would certainly be of interest if the assumption of tautness could be removed from Theorem 2. It is not needed in the top-dimensional case [10]. Some results for the $k=1$ case have been obtained by Fox [8] for general complex manifolds, but with a local tautness assumption.

THEOREM 3. Suppose that $M$ is an $n$-dimensional complex manifold $(n \geq 2)$ and $p \in M$. Suppose that there is a point $p \in M$ and an integer $k$, $2 \leq k \leq n$ such that $E_{k}(p ; \alpha)=C_{k}(p ; \alpha) \neq 0$ for all $\alpha \in D_{p}^{k} M$. Then 
$E_{1}(p ; v)=C_{1}(p ; v)$ for all $v \in T_{p} M$ and $I_{K}(p)=I_{C}(p)$ is an analytic ellipsoid.

Proof. The assumptions of Theorem 3 together with Theorem 1 imply that $E_{1}(p ;)=C_{1}(p ;)$, and that whenever $L$ is a $k$-dimensional complex subspace of $T_{p} M$ then $I_{K}(p) \cap L=I_{C}(p) \cap L$ is an analytic ellipsoid. The boundedness of $I_{C}(p)=I_{K}(p)$ follows from the continuity of $C_{1}(p ;)$, and of course $I_{C}(p)$ is always a convex circled domain. We now wish to argue that a bounded convex circled domain in $\mathbf{C}^{n}$ which intersects every $k$-dimensional complex subspace in a $k$-dimensional analytic ellipsoid $(k \geq 2)$ must be an $n$-dimensional analytic ellipsoid. It clearly suffices to establish the result when $k=2$, but in this case our statement is equivalent to the fact that a (finite-dimensional) Banach space in which the parallelogram law holds is a Hilbert space, for the parallelogram law is a statement about the Banach space norm on all two-dimensional subspaces.

THEOREM 4. Suppose that $M$ is an n-dimensional complex manifold $(n \geq 2)$ and $p \in M$. Suppose that there is a point $p \in M$ such that for all $\alpha \in D_{p}^{2} M$ there is a 2-dimensional holomorphic retract of $M$ tangent to $\alpha$ at p. Then if $M$ is Carathéodory-hyperbolic (respectively if $M$ is hyperbolic and $I_{K}(p)$ is convex) then $I_{C}(p)$ is an analytic ellipsoid (respectively $I_{K}(p)$ is an analytic ellipsoid).

Proof. It is easy to see that the hypotheses of the theorem imply that whenever $L$ is a two-dimensional complex subspace of $T_{p} M$ there are (complex) linear retractions of $I_{C}(p)$ onto $I_{C}(p) \cap L$ and of $I_{K}(p)$ onto $I_{K}(p) \cap L$. We now invoke a result of Bohnenblust [3] concerning projections in complex Banach spaces: if $X$ is a complex Banach space in which there is a projection of norm 1 onto any two-dimensional complex subspace, then $X$ is a Hilbert space. (The real case of this result is due to Kakutani [11] and Phillips [16].) Of course the unit ball in any finite dimensional Hilbert space is an ellipsoid (an analytic ellipsoid in the complex case).

THEOREM 5. Suppose that $\Omega$ is a bounded complete circular domain of holomorphy in $\mathbf{C}^{n}, n \geq 2$, and that for some $k, 2 \leq k \leq n$, we have $E_{k}(0 ; \alpha)=C_{k}(0 ; \alpha)$ for all $\alpha \in D_{0}^{k} \Omega$. Then $\Omega$ is an analytic ellipsoid.

Proof. From a result of Barth [1] we obtain the fact that $I_{K}(0)=\Omega$. We now simply invoke Theorem 3 . 
5. The Schwarz lemma on circular domains. A domain $\Omega \subset \mathbf{C}^{n}$ is called circled or circular if $z \in \Omega$ implies $e^{i \theta} \cdot z \in \Omega$ for all real $\theta$. $\Omega$ is complete circular or star-shaped circular or disked if $z \in \Omega$ implies $c z \in \Omega$ for all $c \in \mathbf{C}$ such that $|c| \leq 1 . \Omega$ is convex circled if $\Omega$ is simultaneously circular and convex.

In $\$ 6$ we shall need a version of the Schwarz lemma for mappings from circular domains to convex circled domains (cf. [20, Theorem 8.1.2] for the case when the map is defined on a complete circular domain). The following result actually generalizes the Cauchy estimates; the idea for the proof comes from an argument of Lempert [13, p. 261].

LEMMA 3. Suppose that $\Omega_{1} \subset \mathbf{C}^{n_{1}}$ is circled and contains the origin and $\Omega_{2} \subset \mathbf{C}^{n_{2}}$ is convex circled. Suppose that $f: \Omega_{1} \rightarrow \Omega_{2}$ is a holomorphic map such that $f(0)=0$. Let $f=\sum_{j=1}^{\infty} P_{j}$ be the series expansion of $f$ in terms of ( $n_{2}$-tuples of ) homogeneous polynomials. Then $P_{j}\left(\Omega_{1}\right) \subset \Omega_{2}$.

Proof. We note that

$$
P_{j}(z)=\frac{1}{2 \pi} \int_{0}^{2 \pi} e^{-\imath j \theta} f\left(z e^{\imath \theta}\right) d \theta
$$

and that the series expansion of $f$ is valid for all $z \in \Omega_{1}[\mathbf{5}, \mathrm{p}$. 17]. Now fix $z \in \Omega_{1}$. The set $\left\{f\left(z e^{i \theta}\right) \mid \theta \in \mathbf{R}\right\}$ is a compact subset $K_{1}$ of $\Omega_{2}$. Let $K_{2}$ be the circled subset of $\Omega_{2}$ generated by $K_{1}$ (also compact), and let $\hat{K}_{2}$ be its convex hull, a convex circled set and a compact subset of $\Omega_{2}$. Now any value of the integrand in (5.1) lies in $K_{2}$, and any Riemann sum for the right-hand side of (5.1) is a convex combination of points in $K_{2}$. Hence $P_{j}(z) \in \hat{K}_{2} \subset \Omega_{2}$.

6. Intrinsic measures on circular domains. Using the lemmas in $\S 3$, we show how to compute the Eisenman norm of $k$-vectors at the origin of a complete circular domain of holomorphy. For the Carathéodory norms we consider slightly more general domains (circular domains containing 0), but unless $k=1$ or $k=n$ we obtain only a pair of inequalities, essentially because of the nonexistence in general of projections of norm 1 onto subspaces of a Banach space of dimension greater than one.

THEOREM 6. Let $\Omega$ be a complete circular domain of holomorphy. Let $\alpha \in D_{0}^{k} \Omega$ and let $L$ be the $k$-dimensional complex linear subspace of $\mathbf{C}^{n}$ tangent to $\alpha$ at 0 . Let $s$ be the supremum of the volumes of the $k$-dimensional analytic ellipsoids (centered at 0 as always) which can be inscribed in 
$L \cap \Omega$. Then

$$
E_{k}(0 ; \alpha)=\|\alpha\|^{2} \operatorname{vol}\left(B_{k}\right) s^{-1} \text {. }
$$

( $\|\alpha\|$ and $s$ may be computed using the Euclidean metric or any constantcoefficient hermitian metric.)

Proof. The inequality $E_{k}(0 ; \alpha) \geq\|\alpha\|^{2} \operatorname{vol}\left(B_{k}\right) s^{-1}$ follows from Lemma 2 and the fact, already noted, that $I_{K}(0)$ coincides with $\Omega[1]$. The opposite inequality is obtained by considering complex linear maps from $B_{k}$ into $\Omega \cap L$.

REMARKs. (1) A linear extremal map exists if $\Omega$ is bounded (cf. Lemma 2).

(2) Remark 1 shows that an extremal map for $\alpha \in D_{0}^{k} \Omega$ need not be proper unless $\Omega \cap L$ is a $k$-dimensional analytic ellipsoid. This is to be contrasted with the behaviour of extremal mappings for the Kobayashi metric on strictly convex domains [12].

In studying the Carathéodory norms of $k$-vectors at the origin of a circular domain, we first note the following:

Lemma 4. Let $\Omega$ be a circular domain in $\mathbf{C}^{n}$ and let $\hat{\Omega}$ denote its convex hull, a convex circled domain. Then

$$
C_{k}^{\Omega}(0 ;)=C_{k}^{\hat{\Omega}}(0 ;) \quad k=1, \ldots, n .
$$

Proof. From Lemma 3 we see that it suffices to consider linear maps from $\Omega$ to $B_{k}$ in computing $C_{k}^{\Omega}(0 ;)$ and $C_{k}^{\hat{\Omega}}(0 ;)$. But if $T: \mathbf{C}^{n} \rightarrow \mathbf{C}^{k}$ is any linear map such that $T(\Omega) \subset B_{k}$, then from the fact that $T(\hat{\Omega})=\widehat{T(\Omega)}$ it follows that $T(\hat{\Omega}) \subset B_{k}$. Hence the class of linear maps under consideration is identical whether one is computing $C_{k}^{\Omega}(0 ; \alpha)$ or $C_{k}^{\hat{\Omega}}(0 ; \alpha)$.

THEOREM 7. (a) Let $\Omega$ be a circular domain in $\mathbf{C}^{n}$ and let $\hat{\Omega}$ be its convex hull. Let $\alpha \in D_{0}^{k} \Omega$ and let $L$ be the $k$-dimensional linear subspace of $\mathrm{C}^{n}$ tangent to $\alpha$ at 0 . Let $t$ be the infimum of the volumes of the $k$-dimensional analytic ellipsoids in $L$ which contain $L \cap \hat{\Omega}$ ( $t$ is finite iff $L \cap \hat{\Omega}$ is bounded, in which case $t$ is assumed.) Then

$$
C_{k}(0 ; \alpha) \leq\|\alpha\|^{2} \operatorname{vol}\left(B_{k}\right) t^{-1} \text {. }
$$


(b) If in addition $\Omega$ is bounded let $m$ denote the minimal norm with respect to the Banach space structure on $\mathbf{C}^{n}$ whose unit ball is $\hat{\Omega}$ of $a$ projection of $\mathbf{C}^{n}$ onto L. (Thus $m \geq 1$.) Then

$$
m^{-2 k}\|\alpha\|^{2} \operatorname{vol}\left(B_{k}\right) t^{-1} \leq C_{k}(0 ; \alpha) \leq\|\alpha\|^{2} \operatorname{vol}\left(B_{k}\right) t^{-1} \text {. }
$$

$\left(\|\alpha\|^{2}\right.$ and $t$ may be computed using the Euclidean metric or any constantcoefficient hermitian metric; $\operatorname{vol}\left(B_{k}\right)$ denotes the Euclidean volume of the unit ball in $\mathbf{C}^{k}$.)

Proof. (a) The right-hand side of (6.3) is zero iff $L \cap \hat{\Omega}$ is unbounded. In this case there does not exist a linear map $T: \hat{\Omega} \rightarrow B_{k}$ such that $T_{*}(\alpha) \neq 0$. In view of Lemmas 3 and 4 it follows that if $g: \Omega \rightarrow B_{k}$ is any holomorphic map such that $g(0)=0$ then $g_{*}(\alpha)=0$. Hence $C_{k}(0 ; \alpha)=0$ and (6.3) is valid in this case.

We may therefore assume that $L \cap \hat{\Omega}$ is bounded. For the fact that the infimum $t$ is assumed in this case see Lemma 2. In view of Lemma 3 it suffices to consider linear maps $T: \Omega \rightarrow B_{k}$ in estimating $C_{k}^{\Omega}(0 ; \alpha)$. Since $T(L \cap \hat{\Omega}) \subset B_{k}$ for such a map we obtain, abbreviating $\left(\left.T\right|_{L}\right)_{*}^{-1}$ to $T_{*}^{-1}$,

$$
\frac{\left\|T_{*}(\alpha)\right\|^{2}}{\|\alpha\|^{2}}=\frac{\operatorname{vol}\left(B_{k}\right)}{\operatorname{vol}\left(T_{*}^{-1}\left(B_{k}\right)\right)} \leq \frac{\operatorname{vol}\left(B_{k}\right)}{t}
$$

since $T_{*}^{-1}\left(B_{k}\right)$ is an analytic ellipsoid containing $L \cap \hat{\Omega}$.

(b) To prove the remaining inequality in (b), let $A$ be an analytic ellipsoid of minimal volume circumscribing $L \cap \hat{\Omega}$ and let $T: L \rightarrow \mathrm{C}^{k}$ be a linear transformation such that $T(A)=B_{k}$. Let $\pi$ : $\mathbf{C}^{n} \rightarrow L$ be a projection of minimal norm $m \geq 1$ with respect to the Banach space structure on $\mathbf{C}^{n}$ whose unit ball is $\hat{\Omega}$. It is elementary to see that a projection of minimal norm exists, and also, in view of the linearity of the transformations, that $m^{-1} \cdot T \circ \pi$ maps $\Omega$ into $B_{k}$. Hence

$$
\begin{aligned}
\frac{\left\|\left(m^{-1} \cdot T \circ \pi\right)_{*}(\alpha)\right\|^{2}}{\|\alpha\|^{2}} & =\frac{\left\|\left(m^{-1} \cdot T\right)_{*}(\alpha)\right\|^{2}}{\|\alpha\|^{2}} \\
& =m^{-2 k} \frac{\left\|T_{*}(\alpha)\right\|^{2}}{\|\alpha\|^{2}}=m^{-2 k} \operatorname{vol}\left(B_{k}\right) t^{-1}
\end{aligned}
$$

from which it follows that $C_{k}(0 ; \alpha) \geq m^{-2 k}\|\alpha\|^{2} \operatorname{vol}\left(B_{k}\right) t^{-1}$. This completes the proof of Theorem 7 .

REMARK. There always exists a projection of norm 1 onto a 1-dimensional subspace of a Banach space by the Hahn-Banach theorem. It is this fact which allowed Carathéodory [4] to compute the infinitesimal 
Carathéodory metric at the origin of a convex-circled domain. Projections of norm 1 also play a role in the study of fixed points of holomorphic maps [21].

\section{REFERENCES}

[1] T. J. Barth, The Kobayashi indicatrix at the center of a circular domain, Proc. Amer. Math. Soc., 88 (1983), 527-530.

[2] J. Bland and Ian Graham, On the Hausdorff measures associated to the Caratheodory and Kobayashi metrics, Ann. Scuola Norm. Sup. Pisa, 12 (1985), 503-514.

[3] H. Bohnenblust, A characterization of complex Hilbert spaces, Portugaliae Math., 3 (1942), 103-109.

[4] C. Carathéodory, Über die Geometrie der analytischen Abbildungen, die durch analytische Funktionen von zwei Veränderlichen vermittelt werden, Abh. Math. Sem. Univ. Hamburg, 6 (1928), 96-145.

[5] H. Cartan, Les fonctions de deux variables complexes et le problème de la représentation analytique, J. de Math. Pures Appl., 96 (1931), 1-114.

[6] I. M. Dektyarev, Tests for equivalence of hyperbolic manifolds, Funktsional. Anal. i Prilozhen., 15 (1981), 73-74. (English translation: Functional Anal. Appl., 15 (1981), 292-293 (1982).)

[7] D. A. Eisenman (= D. A Pelles), Intrinsic Measures on Complex Manifolds and Holomorphic Mappings, Memoirs Amer. Math. Soc., No. 96, Amer. Math. Soc., Providence 1970.

[8] W. C. Fox, The Reiffen and Royden pseudometrics, to appear.

[9] I. Graham and H. Wu, Some remarks on the intrinsic measures of Eisenman, Trans. Amer. Math. Soc., 288 (1985), 625-660.

[10] I. Graham and $\mathrm{H}$. Wu, Characterizations of the unit ball $B^{n}$ in complex Euclidean space, Math. Z., 189 (1985), 449-456.

[11] S. Kakutani, Some characterizations of Euclidean spaces, Japan J. Math., 16 (1939), 93-97.

[12] L. Lempert, La métrique de Kobayashi et la representation des domaines sur la boule, Bull. Soc. Math. France, 109 (1981), 427-474.

[13] _ Holomorphic retracts and intrinsic metrics in convex domains, Anal. Math., 8 (1982), 257-261.

[14] _ Intrinsic distances and holomorphic retracts, Complex Analysis and Applications '81, Sofia (1984), 341-364.

[15] D. A. Pelles (= D. A. Eisenman), Holomorphic maps which preserve intrinsic measure, Amer. J. Math., 97 (1975), 1-15.

[16] R. S. Phillips, A characterization of Euclidean spaces, Bull. Amer. Math. Soc., 46 (1940), 930-933.

[17] J.-P. Rosay, Sur une caractérization de la boule parmi les domaines de $\mathbf{C}^{n}$ par son groupe d'automorphismes, Ann. l'Inst. Fourier, 29 (1979), 91-97.

[18] H. Rossi, Vector fields on analytic spaces, Ann. Math., 78 (1963), 455-467.

[19] H. L. Royden and P. M. Wong, Carathéodory and Kobayashi metric on convex domains, to appear.

[20] W. Rudin, Function Theory in the Unit Ball of $\mathbf{C}^{n}$, Springer Verlag, Berlin-Heidelberg-New York, 1980.

[21] J.-P. Vigué, Points fixes d'applications holomorphes dans un domaine borné convexe de $\mathbf{C}^{n}$, Trans. Amer. Math. Soc., 289 (1985), 345-353. 
[22] B. Wong, Characterization of the unit ball in $\mathbf{C}^{n}$ by its automorphism group, Invent. Math., 41 (1977), 253-257.

[23] H. Wu, Normal families of holomorphic mappings, Acta Math., 119 (1967), 194-233.

Received July 31, 1986 and in revised form January 6, 1987. Partially supported by the Natural Sciences and Engineering Research Council of Canada.

UNIVERSITY OF TORONTO

TORONTO, CANADA

M5S 1A1 



\section{EDITORS}

\author{
V. S. VARADARAJAN \\ (Managing Editor) \\ University of California \\ Los Angeles, CA 90024 \\ HeRbert Clemens \\ University of Utah \\ Salt Lake City, UT 84112 \\ R. FINN \\ Stanford University \\ Stanford, CA 94305
}

\author{
HERMANN FLASCHKA \\ University of Arizona \\ Tucson, AZ 85721 \\ RAMESh A. GANGOLLI \\ University of Washington \\ Seattle, WA 98195 \\ VAUGHAN F. R. JONES \\ University of California \\ Berkeley, CA 94720
}

\author{
ROBION KIRBY \\ University of California \\ Berkeley, CA 94720 \\ C. C. MOORE \\ University of California \\ Berkeley, CA 94720 \\ HAROLD STARK \\ University of California, San Diego \\ La Jolla, CA 92093
}

\section{ASSOCIATE EDITORS}
R. ARENS
E. F. BECKENBACH
B. H. NEUMANN
F. WOLF
K. YOSHIDA
(1906-1982)

\section{SUPPORTING INSTITUTIONS}

UNIVERSITY OF ARIZONA
UNIVERSITY OF BRITISH COLUMBIA
CALIFORNIA INSTITUTE OF TECHNOLOGY
UNIVERSITY OF CALIFORNIA
MONTANA STATE UNIVERSITY
UNIVERSITY OF NEVADA, RENO
NEW MEXICO STATE UNIVERSITY
OREGON STATE UNIVERSITY

\author{
UNIVERSITY OF OREGON \\ UNIVERSITY OF SOUTHERN CALIFORNIA \\ STANFORD UNIVERSITY \\ UNIVERSITY OF HAWAII \\ UNIVERSITY OF TOKYO \\ UNIVERSITY OF UTAH \\ WASHINGTON STATE UNIVERSITY \\ UNIVERSITY OF WASHINGTON
}

The Supporting Institutions listed above contribute to the cost of publication of this Journal, but they are not owners or publishers and have no responsibility for its content or policies.

Mathematical papers intended for publication in the Pacific Journal of Mathematics should be in typed form or offset-reproduced (not dittoed), double spaced with large margins. Please do not use built up fractions in the text of the manuscript. However, you may use them in the displayed equations. Underline Greek letters in red, German in green, and script in blue. The first paragraph must be capable of being used separately as a synopsis of the entire paper. In particular it should contain no bibliographic references. Please propose a heading for the odd numbered pages of less than 35 characters. Manuscripts, in triplicate, may be sent to any one of the editors. Please classify according to the scheme of Math. Reviews, Index to Vol. 39. Supply name and address of author to whom proofs should be sent. All other communications should be addressed to the managing editor, or Elaine Barth, University of California, Los Angeles, California 90024.

There are page-charges associated with articles appearing in the Pacific Journal of Mathematics. These charges are expected to be paid by the author's University, Government Agency or Company. If the author or authors do not have access to such Institutional support these charges are waived. Single authors will receive 50 free reprints; joint authors will receive a total of 100 free reprints. Additional copies may be obtained at cost in multiples of 50 .

The Pacific Journal of Mathematics is issued monthly as of January 1966. Regular subscription rate: $\$ 190.00$ a year (5 Vols., 10 issues). Special rate: $\$ 95.00$ a year to individual members of supporting institutions.

Subscriptions, orders for numbers issued in the last three calendar years, and changes of address should be sent to Pacific Journal of Mathematics, P.O. Box 969, Carmel Valley, CA 93924, U.S.A. Old back numbers obtainable from Kraus Periodicals Co., Route 100, Millwood, NY 10546.

The Pacific Journal of Mathematics at P.O. Box 969, Carmel Valley, CA 93924 (ISSN 0030-8730) publishes 5 volumes per year. Application to mail at Second-class postage rates is pending at Carmel Valley, California, and additional mailing offices. Postmaster: send address changes to Pacific Journal of Mathematics, P.O. Box 969, Carmel Valley, CA 93924.

PUBLISHED BY PACIFIC JOURNAL OF MATHEMATICS, A NON-PROFIT CORPORATION Copyright (c) 1987 by Pacific Journal of Mathematics 


\section{Pacific Journal of Mathematics}

\section{Vol. 130, No. $2 \quad$ October, 1987}

Bernhard Banaschewski, J. L. Frith and C. R. A. Gilmour, On the congruence lattice of a frame ............................ 209

Paul S. Bourdon, Density of the polynomials in Bergman spaces ........2215

Lawrence Jay Corwin, Approximation of prime elements in division

algebras over local fields and unitary representations of the multiplicative group

Stephen R. Doty and John Brendan Sullivan, On the geometry of extensions of irreducible modules for simple algebraic groups

Karl Heinz Dovermann and Reinhard Schultz, Surgery of involutions

with middle-dimensional fixed point set ....................2 275

Ian Graham, Intrinsic measures and holomorphic retracts ............ 299

John Robert Greene, Lagrange inversion over finite fields . . . . . . . . . . . 313

Kristina Dale Hansen, Restriction to $\mathrm{GL}_{2}(\mathrm{O})$ of supercuspidal

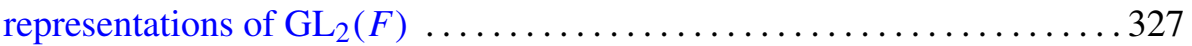

Kei Ji Izuchi, Unitary equivalence of invariant subspaces in the polydisk ....351

A. Papadopoulos and R. C. Penner, A characterization of pseudo-Anosov

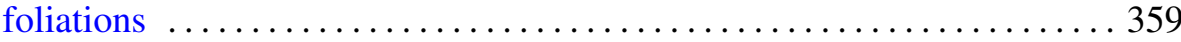

Erik A. van Doorn, The indeterminate rate problem for birth-death

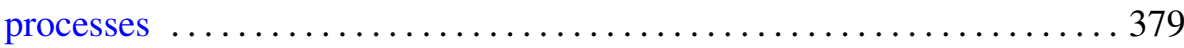

Ralph Jay De Laubenfels, Correction to: "Well-behaved derivations on

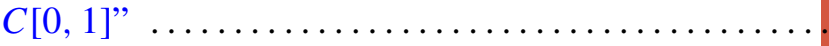

Robert P. Kaufman, Correction to: "Plane curves and removable sets" . . . . 396

Richard Scott Pierce and Charles Irvin Vinsonhaler, Correction to:

"Realizing central division algebras" 